\title{
Neutral Currents in Large Public Lighting Networks
}

\author{
M. Chindris ${ }^{1}$, A. Sudria ${ }^{2}$, A. Cziker ${ }^{1}$, S. Stefanescu ${ }^{1}$ \\ ${ }^{1}$ Electrical Power Systems Department \\ Technical University of Cluj-Napoca \\ 15, C. Daicoviciu St., 400020 Cluj-Napoca (Romania) \\ phone: +400264401451, fax: +40264592055, e-mail: Mircea.Chindris@eps.utcluj.ro \\ ${ }^{2}$ Department of Electrical Engineering \\ E.T.S.I.B., Universidad Politechnica de Catalunya \\ Diagonal 647, 08028 Barcelona (Spain) \\ phone: +34934016727; fax: +34934017433; e-mail: Sudria@citcea.upc.es
}

\begin{abstract}
The modern large public lighting networks are characterized by two major aspects that rise problems from both power quality and neutral current point of view in low voltage wye-connected three-phase electrical systems: (i) the supply system is unbalanced loaded; (ii) the presence of discharge sources. It is important to underline that nowadays, these aspects are not taken into account in the proper sizing of neutral conductors. The paper presents a simplified but enough accurate methodology to estimate the RMS value of neutral currents in lighting networks. The theoretical relationships are illustrated for a three-phase public lighting network that contains high pressure sodium lamps.
\end{abstract}

Key words: unbalance, harmonic pollution, neutral current, public lighting networks

\section{Introduction}

An electrical power system is expected to operate in a balanced three-phase condition. However, a great number of low voltage networks supply three-phase or singlephase asymmetric loads; if the load is unbalanced, the distribution line currents are also unbalanced and cause unequal voltage drop on the distribution lines, such that the load bus voltages are unbalanced. As a result, electrical networks are normally unbalanced, and they have a certain degree of imbalance that depends on the network composition and operation. It is than imperative to conclude that unbalance is a common phenomenon in the distribution system that may be mainly ascribed to the presence of single and double-phase loads and that can cause several quality-related problems; it is however important to realize that unbalance may be created as a result of different operating conditions even within threephase loads $[1,2]$.
On the other hand, the increasing use of non-linear equipment (electronic power converters, saturable devices, arc furnaces and discharge-type lighting) in electrical distribution systems has raised the level of concern about the effects of these loads on the system. Three-phase non-linear loads such as motor drives and other similar devices can create important quality problems but do not contribute significantly to neutral current. Of special concern are single-phase devices with rectifier front-end power supplies (such as computers, electronic lighting ballasts, and other similar electronic devices) or discharge lamps. When these types of loads are connected line to neutral in a three phase wyeconnected power systems, the neutral conductors in the three phase feeders can carry surprising levels of current, even with the loads balanced on the three phases [3, 4].

Taking into account the above mentioned aspects, the possible unbalanced operating conditions resulting in supplying single-phase non-linear loads is of increasing interest because of the proliferation of these types of loads that are widely used [5]. Assuming the three-phase power supply system is symmetrical, the causes of non equal distorted phase current harmonics can be referred, in the most general case, to the type, the number, and the size of the single-phase non-linear loads fed by each phase.

Experience has shown that the harmonic currents do not upset the end-use electronic equipment as much as they overload neutral conductors and transformers. Indeed, the neutral current of a three phase system is given by the summation of the phase currents; these currents at the fundamental and at the harmonics in case of a multigrounded wye distribution system are returned back to the source either through the system neutral or remote 
earth ground. With reference to the neutral current spectrum, in the three-phase system which is perfectly balanced and symmetrical, it contains only triplen order components, because only the triplen harmonic phase currents present zero sequence characteristics. In unbalanced three-phase systems, this is not the case since the phase harmonic currents, including also the fundamental, must be viewed as a superposition of all three symmetrical components: the neutral current spectrum will contain not only triplen harmonics but all the components possessing zero sequence.

Since the national norms has prohibited neutral conductor overcurrent protection, a proper sizing of this conductor is required for the three-phase wye connected distribution systems supplying large number of single-phase nonlinear loads. It is the situation of public lighting systems where, typically, a three-phase four wire distribution network feeds single-phase non-linear loads, namely high pressure discharge lamps.

\section{This paper:}

- Analyzes the unbalance of large public lighting networks;

- Discusses current signature of single phase high intensity discharge lamps and examines the relationship of these signatures to neutral current in wye-connected three-phase electrical systems.

- Establishes formulas for estimating maximum neutral current under various total harmonic current distortion levels for both balanced and unbalanced load conditions.

These discussions apply to $400 / 230 \mathrm{~V}$ electrical systems and lighting distribution systems in Europe.

\section{Unbalance of large public lighting networks}

The majority of light sources in public (street) lighting are HID (High Intensity Discharge) lamps using electromagnetic (classic technology) or electronic ballasts and every lighting grid contains a great number of such single-phase loads. In order to balance the distribution network, they try to connect the same number of lamps on every phase of supply system; however, very often, the whole tree-phase load can appear to the supply network as unbalanced load at the fundamental and at the harmonics.

Let us consider the public lighting network presented in figure 1, where every phase of the distribution system supplies the same number of the same lamps equally spaced out $(z * l$, with $z$ real positive, is the distance from the supply point to the first lamp). In this case, the voltage drop on every line is:

$$
\Delta V^{\alpha}=\sum_{k=1}^{n} \Delta v_{k}^{\alpha} \cdot l_{k}^{\alpha}
$$

where $\alpha$ represents the distribution line;

$n$ - number of lamps on every line;

$l_{k}^{\alpha}$ - distance between the supply point and the lamp $k$ on the line $\alpha$;

$$
\Delta v_{k}^{\alpha}=I_{k}^{\alpha} \cdot\left[r^{\alpha} \cdot \cos \left(\varphi_{k}^{\alpha}\right)+x^{\alpha} \cdot \sin \left(\varphi_{k}^{\alpha}\right)\right] \text { - voltage }
$$
drop on the unity of length produced by the lamp $k$ on the line $\alpha$;

$I_{k}^{\alpha}$ - RMS current absorbed by the lamp $k$ supplied on the $\alpha$ line;

$$
r^{\alpha}, x^{\alpha} \text { - resistance, respectively reactance of }
$$
the $\alpha$ line;

$\varphi_{k}^{\alpha}$ - power factor of the $k$ lamp connected to the $\alpha$ line.

In these conditions, if the distribution system is balanced, the voltage drops can be determined as follows:

$$
\begin{aligned}
& \Delta V^{I}=\Delta v \cdot(2 z+3) \cdot l ; \\
& \Delta V^{I I}=\Delta v \cdot(2 z+5) \cdot l ; \\
& \Delta V^{I I I}=\Delta v \cdot(2 z+7) \cdot l,
\end{aligned}
$$

where $\Delta v=I \cdot(r \cdot \cos \varphi+x \cdot \sin \varphi)$ represents the voltage drop on the line unity of length.

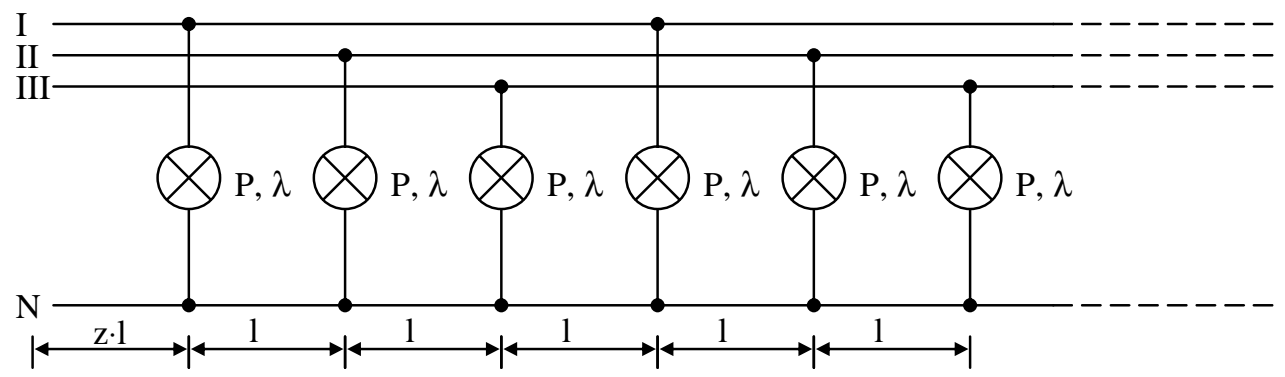

Fig. 1. Public lighting network (unbalanced) 
From the above relationships, it results that even in the hypotheses taken into consideration, the lighting network is unbalanced as the voltage drop on every line is different. If one considers $n$ lamps on every phase, the voltage unbalance factor equals zero-unbalance factor and can be calculated with:

$$
k_{V}^{-}=k_{V}^{0}=\frac{2 \cdot \sqrt{3}}{6 \cdot(z+1)+9(n-1)-\frac{6 \cdot V_{n o m}}{\Delta v \cdot l \cdot n}} .
$$

For the lighting network presented in figure $1, n=2$, and:

$$
k_{V}^{-}=k_{V}^{0}=\frac{3.464}{\left|6 \cdot z+15-\frac{3 \cdot V_{\text {nom }}}{\Delta v \cdot l}\right|} ;
$$

as a result, we can estimate the total unbalance factor [4]:

$$
k_{V}=k_{V}^{-}+k_{V}^{0}=\frac{2 \cdot 3.464}{\left|6 \cdot z+15-\frac{3 \cdot V_{\text {nom }}}{\Delta v \cdot l}\right|} .
$$

Figure 2 presents a new situation where the lamps are unsuitable distributed on the three phases of the supply system; in this case, the voltage drops are:

$$
\begin{aligned}
& \Delta V^{I}=\Delta v \cdot(2 z+1) \cdot l ; \\
& \Delta V^{I I}=\Delta v \cdot(2 z+5) \cdot l ; \\
& \Delta V^{I I I}=\Delta v \cdot(2 z+9) \cdot l .
\end{aligned}
$$

The voltage unbalance is greater as the total unbalance factor has the value:

$$
k_{V}=\frac{2 \cdot 6.928}{\left|6 \cdot z+15-\frac{3 \cdot V_{\text {nom }}}{\Delta v \cdot l}\right|} .
$$

The right solution that guarantees the balance of the lighting network is presented in figure 3; this configuration, known as $U$ distribution, assures the following voltage drops:

$$
\begin{aligned}
& \Delta V^{I}=\Delta v \cdot(2 z+5) \cdot l \\
& \Delta V^{I I}=\Delta v \cdot(2 z+5) \cdot l \\
& \Delta V^{I I I}=\Delta v \cdot(2 z+5) \cdot l
\end{aligned}
$$

i.e. all lines are equally loaded and the total unbalance factor is zero.

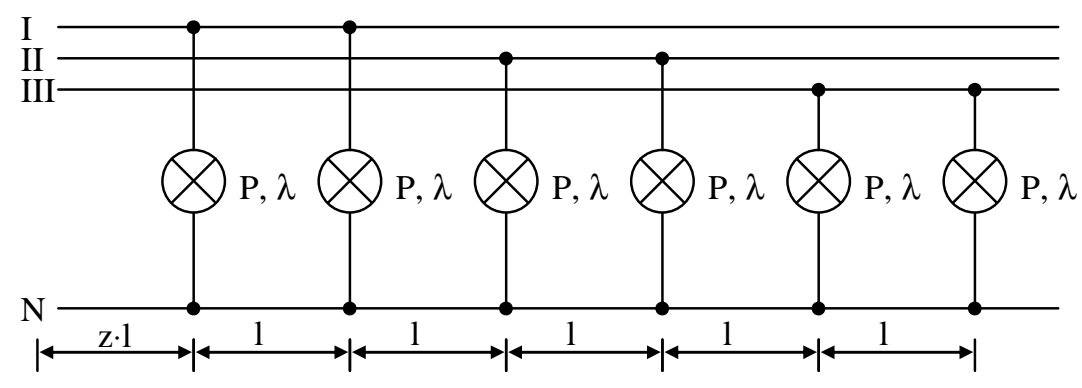

Fig. 2. Public lighting network (unbalanced)

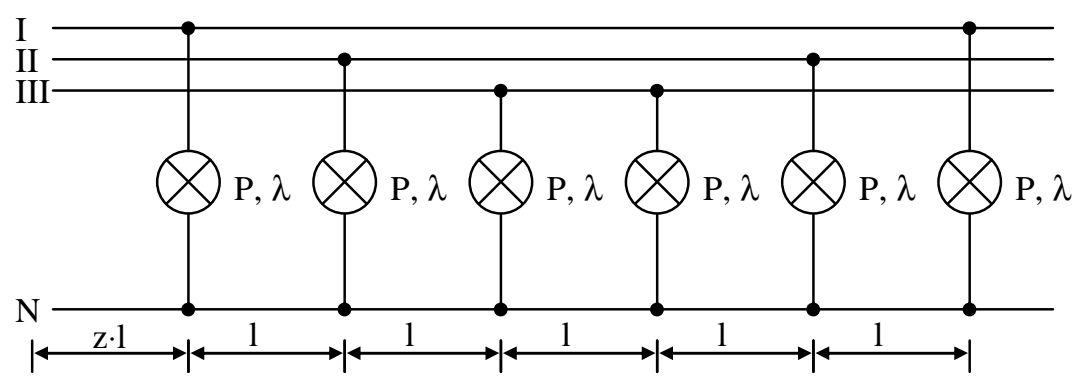

Fig. 3. Public lighting network (balanced)

If the lamps are unequally distributed between the three phases, the electrical distribution system will be unbalanced. In this case, the RMS value of the neutral current can be computed as [6]:

$$
I_{\text {neutral }}=3 I_{0}=3 k_{I}^{0} I_{f}
$$

where $I_{0}$ represents the zero sequence of the line current, $I_{f}$ its RMS value (the positive sequence of the balanced system) while $k_{I}^{0}$ is the zero-unbalance factor of line current.

\section{Non-linear loads and neutral current}

HID lamps are typical single-phase non-linear loads. The measured current spectrum for a high pressure sodium lamp used in street lighting systems is presented in figures $4-7$ and highlights an abundant content of current harmonics, especially after connecting the power factor correction capacitor. 


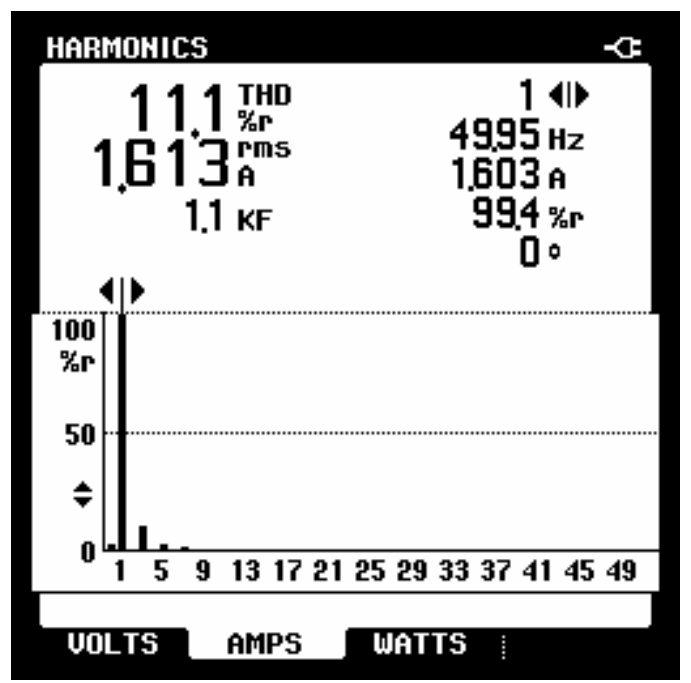

Fig. 4. Harmonic pollution data - $150 \mathrm{~W}$ SON-T lamp without power factor compensation capacitor, full power.

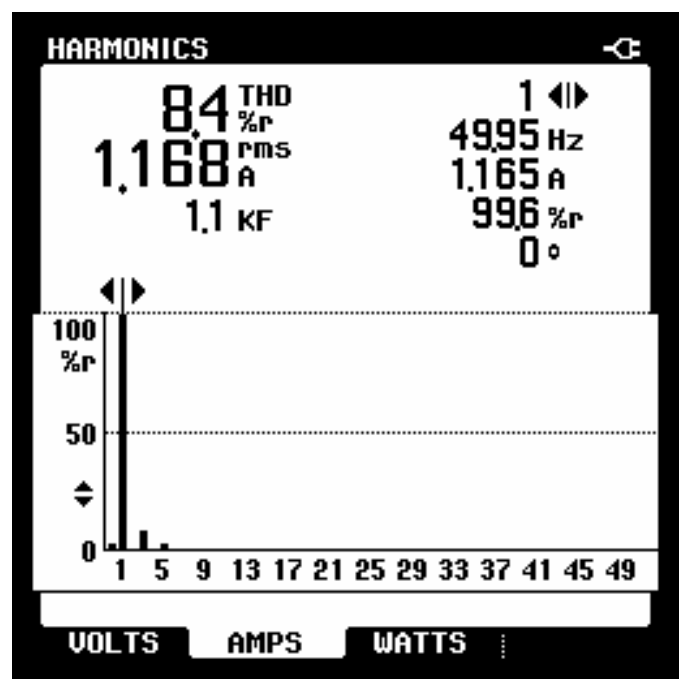

Fig. 6. Harmonic pollution data - $150 \mathrm{~W}$ SON-T lamp without power factor compensation capacitor, dimmed.

It is well known that in the case of 4 wires distribution networks supplying non-linear loads, an important effect of the harmonic pollution is a supplementary neutral current; with reference to the neutral current spectrum, in three phase systems which are perfectly balanced and symmetrical, it contains only triplen order components, because only the triplen harmonic phase currents present zero sequence characteristics. In such conditions, the instantaneous value of the neutral current can be written as

$$
i_{\text {neutral }}=3 \sum_{k=1}^{\infty} I_{3 k} \cdot \sin \left(3 \cdot k \cdot \omega \cdot t+\beta_{k}\right)
$$

and has the RMS value

$$
I_{\text {neutral }}=3 \cdot \sqrt{\sum_{k=1}^{\infty} I_{3 k}^{2}},
$$

where $I_{3 k}$ is the RMS value of the $3 k$ harmonic current. Usually, unfiltered single phase non-linear loads like gas discharge lamps produce current distortions that contain large amounts of $3^{\text {rd }}, 5^{\text {th }}, 7^{\text {th }}, 9^{\text {th }}, \ldots, 15^{\text {th }}$ harmonic and so

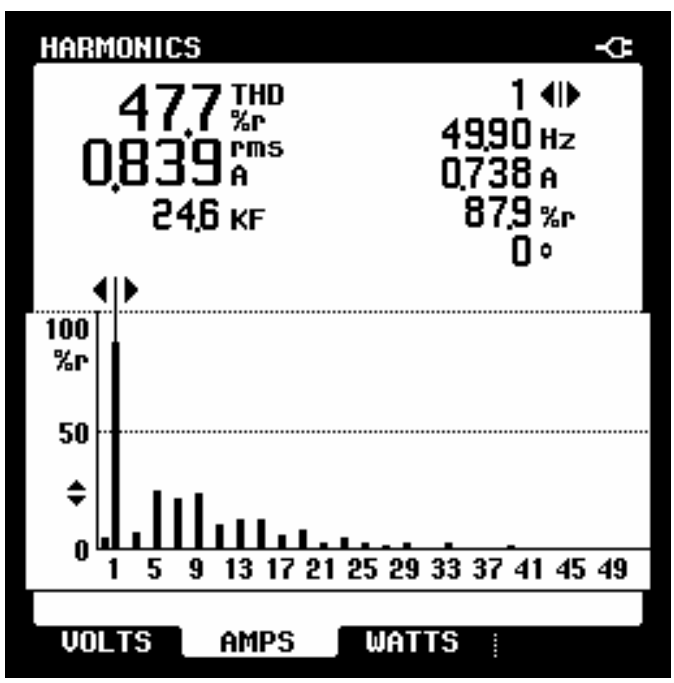

Fig. 5. Harmonic pollution data - $150 \mathrm{~W}$ SON-T lamp with power factor compensation capacitor, full power.

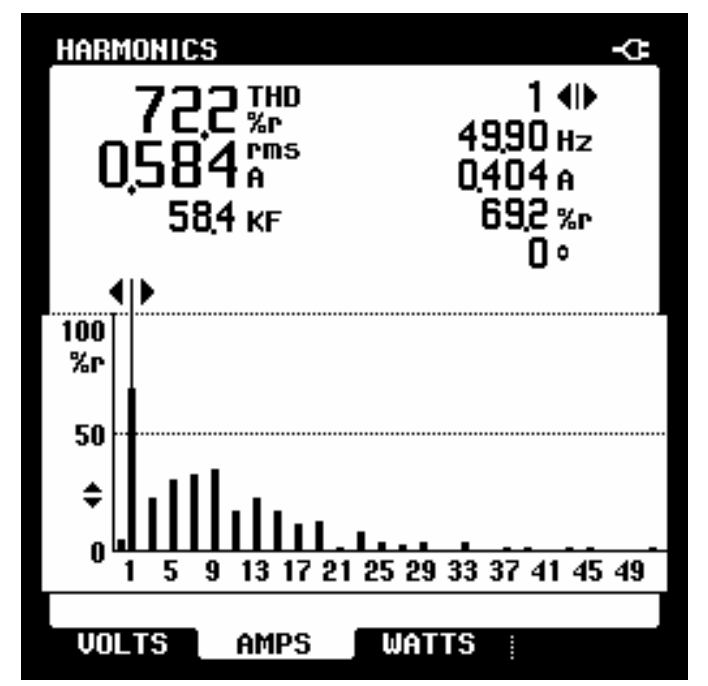

Fig. 7. Harmonic pollution data $-150 \mathrm{~W}$ SON-T lamp with power factor compensation capacitor, dimmed.

on. Of those, only the multiple of three harmonics like $3^{\text {rd }}$, $9^{\text {th }}, 15^{\text {th }}$, etc contribute to the neutral problem; normally, because of their lower current levels, the $15^{\text {th }}$ and higher triplen harmonics do not significantly affect the RMS neutral current value. Therefore, in case of a distribution system supplying 3 single-phase non-linear loads, a sufficiently accurate estimation of the neutral current, $\mathrm{I}_{\text {neutral }}$, is obtained by multiplying with three the higher triplen current harmonic value [7]

$$
I_{\text {neutral }} \approx 3 \cdot I_{3 k} \text {. }
$$

This approach allows the following commentary: a percentage of $33 \%$ for a triplen harmonic current, with respect to the phase current, leads to a $100 \%$ additional overload of the neutral wire.

The RMS phase current value, including harmonic current, can be obtained from individual current harmonics, as 


$$
I_{f}=\sqrt{\sum_{k=1}^{\infty} I_{k}^{2}}[\mathrm{~A}]
$$

The formula for the current total harmonic distortion is defined by

$$
T H D_{i}=\frac{I_{d}}{I_{1}} \cdot 100=\frac{\sqrt{\sum_{k=2}^{\infty} I_{k}^{2}}}{I_{1}} \cdot 100=\sqrt{\sum_{k=2}^{\infty} \frac{I_{k}^{2}}{I_{1}^{2}}} \cdot 100[\%],
$$

where $I_{k}$ is the RMS value of the $k^{\text {th }}$ harmonic current, and $I_{l}$ is the RMS value of the fundamental current.

If we denote with $\gamma_{k}$, the level of the $k^{\text {th }}$ current harmonic

$$
\gamma_{k}=\frac{I_{k}}{I_{1}} \cdot 100[\%]
$$

relationship (14) becomes

$$
T H D_{i}=\sqrt{\sum_{k=2}^{\infty} \gamma_{k}^{2}}[\%] .
$$

Equations (14) and (16) indicate that, without harmonics, the total RMS current is simply the value of the fundamental component that is, the total harmonic distortion $\left(\mathrm{THD}_{\mathrm{i}}\right)$ of a sinusoid current waveform, is 1 .

On the other hand, from (13) and (14) it results:

$$
I_{1}=\frac{I_{f}}{\sqrt{1+\left(T H D_{i}\right)^{2}}},[\mathrm{~A}],
$$

respectively

$$
\frac{I_{\text {neutral }}}{I_{f}}=\frac{3 \cdot \sqrt{\sum_{k=1} \gamma_{3 k}^{2}}}{\sqrt{1+\left(T H D_{i}\right)^{2}}} .
$$

If we consider only the greater harmonic current, from the above relationships we can write:

$$
\frac{3 \cdot \gamma_{\max }}{\sqrt{1+\left(T H D_{i}\right)^{2}}} \leq b_{\text {harm }} \leq \frac{3}{\sqrt{1+\left(T H D_{i}\right)^{2}}}
$$

where

$\gamma_{\max }$ represents the level of the most important triplen harmonic;

$$
b_{\text {harm }}=\frac{I_{\text {neutral }}}{I_{f}} .
$$

Figure 8 represents the values of the coefficient $b_{\text {harm }}$ as a function of the line current total harmonic distortion (as parameter is taken the level of the most important current harmonic).

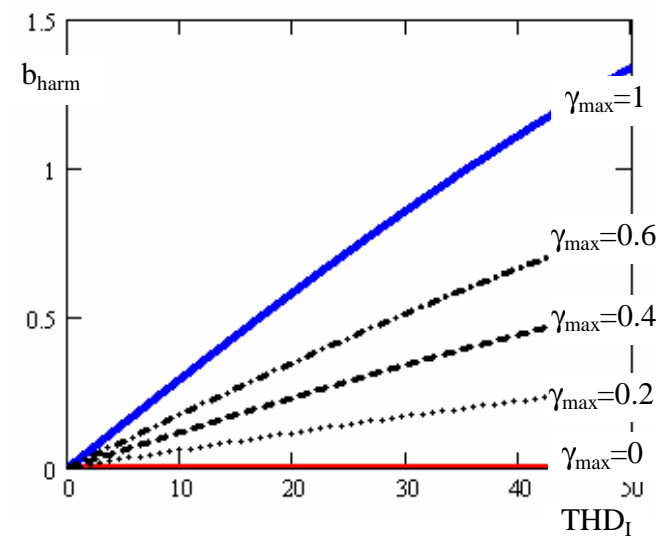

Fig. 8. The range of the parameter $b_{\text {harm }}$ as a function of line current total harmonic distortion

\section{Neutral current in unbalanced harmonic polluted networks}

In the most disadvantageous circumstances, namely unbalanced and non-sinusoidal conditions, the value of the neutral current will satisfy the relationship:

$$
I_{\text {neutral }}=\left(\frac{3}{\sqrt{1+\left(\frac{100}{T H D_{i}}\right)^{2}}}+3 \cdot k_{I}^{0}\right) \cdot I_{f}
$$

that can be also written as

$$
b_{\text {total }}=\frac{3}{\sqrt{1+\left(\frac{100}{T H D_{i}}\right)^{2}}}+3 \cdot k_{I}^{0}
$$

and is represented in figure 9.

\section{Measurement and computed results}

In order to analyze the behavior of the modern public lighting systems, the authors have implemented the above proposed algorithm for calculating the neutral current in a distribution network supplying a street lighting system. The system has 40 modern luminaries/phase; each luminaries is fitted with a $150 \mathrm{~W}$ SON-T lamp, a capacitor for power factor compensation and a digital power switch for dimming.

The harmonic pollution for the considered high pressure sodium lamp depends on the structure of the circuit: without the compensation capacitor the lamp has a satisfactory harmonic behavior $(\mathrm{THD}=11.1$ - case 1 and $\mathrm{THD}=8.4$ - case 3 ); when the compensation capacitor is added, the harmonic performance is strongly depreciated (THD=47.7 - case 2 and $\mathrm{THD}=72.2$ - case 4) and highlights possible resonance events.

Analyze was performed in two cases (balanced and unbalanced network); for each case, the influence of the 
line current harmonic content was considered by relationship (11) - column $a$, respectively (12) - column $b$. The numerical results are presented in Table 1 , where $\mathrm{d}_{i}$ is the neutral current total harmonic distortion, and show that even when the harmonic pollution is at low level, the neutral current can be great enough if the luminaries are asymmetrically distributed on the three phases of the supply network. For systems characterized as polluted, the neutral current can exceed the line current.
For a balanced system, the neutral current growth due to non-sinusoidal operation can be evaluated in different ways. Depending on the harmonic pollution characteristics, adequate equations can be used: relationship (12) is a simple and quite accurate way to estimate the neutral current when the line current has a dominant triplen harmonic; when there are more components with an important harmonic level, equation (11) is recommended. Even not so exactly, equation (18) allows a quick estimation of the harmonic current contribution to the neutral current.

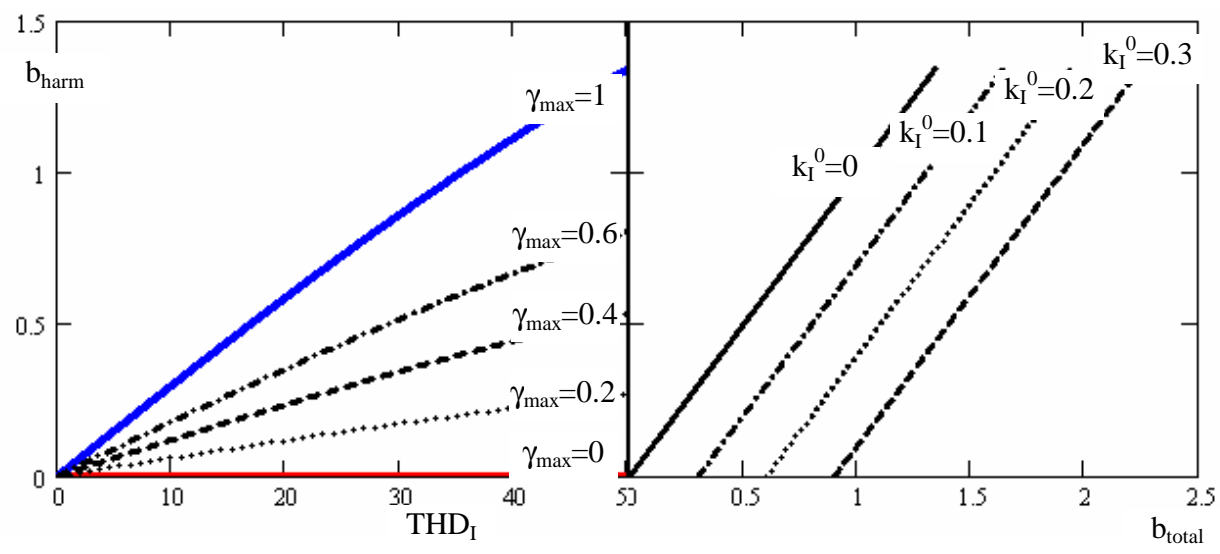

Fig. 9. The range of the parameter $b_{\text {total }}$ as a function of line current total harmonic distortion and zero-unbalance factor of line current

TABEL 1. Computed results

\begin{tabular}{|c|c|c|c|c|c|c|c|c|c|c|}
\hline \multirow[t]{2}{*}{ Case } & \multirow{2}{*}{$\begin{array}{c}\mathrm{I}_{3} \\
{[\mathrm{~A}]}\end{array}$} & \multirow{2}{*}{$\begin{array}{c}\mathrm{I}_{9} \\
{[\mathrm{~A}]}\end{array}$} & \multirow{2}{*}{$\begin{array}{l}\mathrm{I}_{15} \\
{[\mathrm{~A}]}\end{array}$} & \multirow{2}{*}{$\begin{array}{c}d_{i} \\
{[\%]}\end{array}$} & \multirow{2}{*}{$\begin{array}{c}\mathrm{I}_{\mathrm{f}} \\
{[\mathrm{A}]}\end{array}$} & \multicolumn{2}{|c|}{$\begin{array}{c}\mathrm{I}_{\text {neutral }}[\mathrm{A}] \\
\mathrm{k}_{\mathrm{I}}^{0}=0\end{array}$} & \multicolumn{2}{|c|}{$\begin{array}{l}\mathrm{I}_{\text {neutral }}[\mathrm{A}] \\
\mathrm{k}_{\mathrm{I}}^{0}=0.15\end{array}$} & \multirow[t]{2}{*}{ Fig. } \\
\hline & & & & & & $\mathrm{a}$ & $\mathrm{b}$ & $\mathrm{a}$ & $\mathrm{b}$ & \\
\hline 1 & 6.96 & 0 & 0 & 10.86 & 64.52 & 20.89 & 20.89 & 49.93 & 49.93 & 4 \\
\hline 2 & 3.91 & 0 & 0 & 8.4 & 46.72 & 11.74 & 11.74 & 32.75 & 32.75 & 6 \\
\hline 3 & 3.63 & 11.8 & 4.08 & 47.7 & 44 & 39 & 35.39 & 55.67 & 52.46 & 5 \\
\hline 4 & 5.67 & 8.43 & 4.38 & 68.5 & 23.36 & 33.2 & 23.36 & 50.1 & 43 & 7 \\
\hline
\end{tabular}

\section{Conclusions}

Modern luminaries used in public lighting systems are often fitted with high-pressure sodium lamps. These lamps are characterized as non-linear loads and pollute the electrical distribution system. The paper presents some relationships that allow the estimation of the upper limit of the neutral current amplification due to harmonic pollution presence and unbalance.

As a general conclusion, the estimation of the neutral current in electrical public lighting distribution systems is compulsory in order to avoid incidental outrunning of the neutral conductor current capacity.

\section{References}

[1] Chindris,M. Symmetrizing Systems for Single-Phase High-Power Receivers. Socrates Course. April 2001, University of Zaragoza, Spain.

[2] Zheng,T. et all. "Evaluating Power System Unbalance in the Presence of Harmonic Distortion", IEEE Trans. On Power Delivery, vol. 18, No. 2, pp. 393 - 397), April 2003.
[3] Arthur,R. and Shanahan,R.A. "Neutral Currents in Three Phase Wye Systems", in Proc. of ICHQP'96, $7^{\text {th }}$ International Conference on Harmonics and Quality of Power, Las Vegas, October 16-18, 1996, pp. 40 - 47.

[4] Verde,P. et all. "On the Neutral Current Spectra of Thyristor Controlled Incandescent Lighting Loads", in Proc. of ICHQP'9, $7^{\text {th }}$ International Conference on Harmonics and Quality of Power, Las Vegas, October 1618,1996 , pp. $565-569$.

[5] ***PE143/94. "Romanian Standard for Limitation of Unbalance and Non-sinusoidal Operation of Power Systems".

[6] Cziker,A. and Chindris,M. Mitigation of load unbalance, MEDIAMIRA Publishing House, Cluj-Napoca (2003).

[7] Chindris,M. et all. Harmonic pollution mitigation in industrial networks, MEDIAMIRA Publishing House, Cluj-Napoca (2003). 